Methods/Design We compared the number of visits for the last 12 months against the previous 12 months. We obtained data from Wordpress and Podbean platforms for the blog and podcast respectively. We did not add data from other social media platforms such as Twitter and Facebook. Differences are expressed as a ratio to indicate an increase or decrease in the number of engagements from the previous year.

Results/Conclusions The data shows similar levels of between year activity from July 2018 through to February 2019. It then shows a rapid and profound increase in activity from March 2019, coinciding with the rapid spread of the pandemic in Europe and an early blog/podcast with colleagues from Northern Italy. Since then activity has fallen but still remains roughly 1.5 times higher than in previous years. Combined visits to the blog and podcast peaked at 162,019 engagements, representing a near 6.9 fold increase in podcast engagement and a 2.7 increase in blog activity during May 2019.

The COVID-19 pandemic resulted in a rapid and sustained engagement with the St Emlyn's platform. The reasons for this are unclear from the data presented here, but we believe reflect the superior agility of modern educational tools such as blogs and podcasts to share and disseminate information as compared to traditional academic publishing methods.

\section{EARLY SENIOR ASSESSMENT AND ACCESS TO CT IN AN EMERGENCY DEPARTMENT}

${ }^{1}$ Marta de Andres Crespo, ${ }^{2}$ Cheryl Zogg, ${ }^{3}$ Alex Novak, ${ }^{1}$ David Metcalfe. ${ }^{1}$ University of Oxford; ${ }^{2}$ Yale University; ${ }^{3}$ Oxford University Hospitals NHS Foundation Trust

10.1136/emj-2020-rcemabstracts. 10

Aims/Objectives/Background The Rapid Assessment and Treatment (RAT) model provides early senior assessment of undifferentiated 'majors' patients and has been proposed as a strategy for improving Emergency Department (ED) efficiency. One goal of RAT is to organise essential imaging at an earlier stage within the patient's ED journey. This study aimed to identify any potential early impact of a RAT initiative on time to imaging for patients requiring CT head.

Methods/Design Electronic health record data were extracted for all patients that underwent head CT while in the ED over a 54-month period (48 months pre-intervention and 6 postintervention) at a single Major Trauma Centre in England. Interrupted time series analysis was used to estimate any effect of RAT on time from ED arrival to imaging.

Results/Conclusions There was a pre-existing gradual trend over the entire time series towards patients waiting less time for CT. Although time to CT appeared to increase when the RAT model was implemented, this change was small and not statistically significant (9.8 [95\% CI -1.6 to 21.3] minutes). Following RAT implementation, the pre-existing trend towards quicker access to CT resumed but without any change in the slope of the line.

This early evaluation did not identify an association between RAT implementation and speed of access to CT head. The system may mature over time and further evaluations will be necessary to identify delayed effects on access to imaging as well as other process measures intended to improve ED safety and efficiency.

\section{PENTHROX: A SERVICE EVALUATION FOR METHOXYFLURANE USE IN AN EMERGENCY DEPARTMENT SETTING}

${ }^{1}$ Jack Ingham, ${ }^{2}$ Gareth Roberts. ${ }^{1}$ Manchester Foundation Trust; ${ }^{2}$ MFT

10.1136/emj-2020-rcemabstracts. 11

Aims/Objectives/Background In Manchester Royal Infirmary, a major trauma centre, there are many patient presentations which require fast acting analgesia. Often these presentations, such as shoulder dislocations, require conscious sedation in order to be safely, effectively and humanely treated.

Penthrox was introduced to the emergency department in December 2019. It was hoped that it would be used as an alternative to conscious sedation in patients requiring procedures. It was postulated that by having another analgesic method available, patients would have a greater likelihood of being discharged without the requirement for conscious sedation. This would therefore not require waiting for a Resus bed and therefore reduce length of stay in the department.

Methods/Design Data was prospectively gathered for patients who received Penthrox in the months of December 2019 to July 2020 using a questionnaire that was filled in by clinicians.

Audit forms were collated as well as attendance data (including length of stay) for shoulder dislocations Dec 19 Feb 19.

Pain scores were subjectively scored out of 10 and, using ttests, compared for significance.

Length of stay, checklist completion, progression to concious sedation, further pain relief requirements and indications were also analysed.

Results/Conclusions Subjectively reported pain scores were significantly reduced when comparing before and during procedure $(<0.001,95 \%$ CI of a reduction of 1.959 to 5.113$)$ and also before and 10 mins after procedure $(\mathrm{p}=<0.001,95 \%$ CI 3.96 to 6.33 ). The distributions of pain scores are demonstrated in the violin plot below.

There was no significant association between checklist completion and progression to concious sedation.

Difference between length of stay did not quite meet statistical significance $($ mean $=163.62$ using penthrox, mean $=$ 225 without penthrox. $\mathrm{p}=0.06$ ).

There were no significant adverse events.

In conclusion Penthrox is a safe and welcome addition to the ED formulary.

\section{EMERGENCY DEPARTMENT CLINICAL LEADS' EXPERIENCES OF IMPLEMENTING PRIMARY CARE SERVICES WHERE GPS WORK IN OR ALONGSIDE EMERGENCY DEPARTMENTS IN THE UK: A QUALITATIVE STUDY}

${ }^{1}$ Michelle Edwards, 'Alison Cooper, ${ }^{1}$ Freya Davies, 'Andrew Carson-Stevens, ${ }^{2}$ Thomas Hughes, ${ }^{1}$ Alison Porter, ${ }^{3}$ Helen Snooks, ${ }^{1}$ Adrian Edwards. ${ }^{1}$ Cardiff University; ${ }^{2} J o h n$ Radcliffe Hospital; ${ }^{3}$ Swansea University

\subsection{6/emj-2020-rcemabstracts. 12}

Aims/Objectives/Background We aim to describe ED clinical leads' experiences of implementing and delivering 'primary care services' and 'emergency medicine services' where GPs were integrated into the ED team. NHS England policy has promoted services in which patients presenting to EDs with 
non-urgent problems are directed to general practitioners (GPs) and other primary care clinicians working within or alongside EDs to address increasing demand. Our findings describe variation in the ways that these primary care services are implemented and the ways in which GPs work within them. We also highlight successes and challenges in implementing such services.

Methods/Design We conducted interviews with ED clinical leads in England $(n=19)$ and Wales $(n=2)$. We used framework analysis to analyse interview transcripts and explore differences across 'primary care services', 'emergency medicine services' and emergency departments without primary care services.

Results/Conclusions In EDs with separate primary care services, success was reported with a distinct workforce of primary care clinicians, who improved waiting times and flow by seeing primary care-type patients in a timely way, using fewer investigations, and enabling ED doctors to focus on more acutely unwell patients. Some challenges were: aligning services with the policy guidance, inconsistent demand for primary care, accessible community primary care services, difficulties recruiting GPs, lack of funding, difficulties agreeing governance protocols and establishing effective streaming pathways. Where GPs were integrated into an ED workforce success was reported as managing the demand for both emergency and primary care and reducing admissions.

To support successful and sustainable primary care services in or alongside EDs, policy makers and commissioners should consider varied ways that GPs can be employed to manage local demand and also local contextual factors such as the ability to recruit and retain GPs, sustainable funding, clear governance frameworks, training, support and guidance for all staff.

\section{PATIENTS'MOTIVATIONS AND EXPECTATIONS WHEN SEEKING URGENT CARE AT EMERGENCY DEPARTMENTS AND ACCEPTABILITY OF PRIMARY CARE STREAMING: A REALIST STUDY}

'Michelle Edwards, 'Delyth Price, 'Julie Hepburn, 'Barbara Harrington, ${ }^{2}$ Bridie Evans, ${ }^{1}$ Alison Cooper, ${ }^{1}$ Adrian Edwards. ${ }^{1}$ Cardiff University; ${ }^{2}$ Swansea University

\subsection{6/emj-2020-rcemabstracts. 13}

Aims/Objectives/Background We aim to explain the contexts and mechanisms that influence patients' motivations and expectations when accessing urgent care at an ED and their acceptability of being streamed to a primary care clinician working in or alongside the ED. Recent healthcare policy has encouraged the implementation of primary care services in or alongside emergency departments whereby patients with low acuity illness are streamed to a primary care clinician after a brief initial assessment. Our findings describe patients' motivations, expectations, and acceptability of primary care streaming and their level of satisfaction.

Methods/Design We recruited 24 patients to be interviewed after visiting an emergency department for one of five low acuity complaints. 12 patients were streamed to ED clinicians and 11 were streamed to primary care clinicians. We carried out semi-structured realist style interviews by telephone and carried out a realist analysis to create theories to explain motivations to attend, acceptability of streaming and satisfaction with care.
Results/Conclusions Motivations for attending the ED included patients' perception of their complaint as an emergency which needed immediate treatment, and previous experience of receiving care at the ED. Acceptability of primary care streaming was related to patients' past experiences accessing primary care services, their trust in initial assessment processes and their expectation to be seen by 'expert clinicians' on the 'same day'. When patients' expectations of waiting times, level of investigations and general quality of care were met or exceeded, they reported acceptability to being streamed to a primary care clinician and were satisfied with their care. Understanding why patients attend the ED for urgent care needs and their experience of primary care streaming is essential to addressing increasing ED demand and improving efficiency.

\section{RCEM lightning}

\section{CAN NEWS2 BE USED TO PREDICT A\&E RE- ATTENDANCES DURING THE COVID-19 PANDEMIC?}

Krishna Prasad Gopalakrishna Pillai, Zeyar Win Naing, Hemavathi Muniswamy, Abdul Sajjad Pathan. Luton and Dunstable University Hospital

\subsection{6/emj-2020-rcemabstracts. 14}

Aims/Objectives/Background The NEWS2 (National Emergency Warning Score 2) is a widely used tool in Emergency Departments (ED) to identify patients who may be at increased risk of deterioration. NEWS2 is based on a simple aggregate scoring system in which a score is allocated to physiological measurements, already recorded in routine practice, when patients present to, or are being monitored in hospital. Currently there are no predictive scoring systems to evaluate ED re-attendances. The objective of this study was to investigate whether calculated NEWS2 had any influence on the ED re-attendances during the COVID-19 pandemic.

Methods/Design Data for the study was compiled from Symphony, the department's electronic patient records. Data was retrospectively compiled for the month of April 2020 and was subsequently followed up to 28 days for re-attendances. Patients aged 18 years and older, presenting with COVID-19 symptoms who were discharged from ED were only included $(n=310)$. Case definitions for COVID-19 symptomatology were in accordance to the Public Health England guidance.

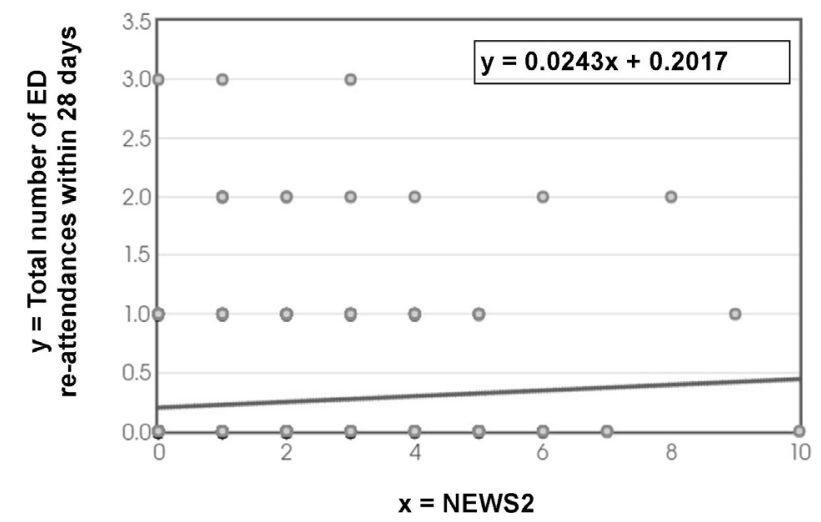

Abstract 301 Figure 1 